\title{
Two-loop five-point integrals in massless QCD
}

\section{Thomas Gehrmann}

Department of Physics, University of Zürich, Winterthurerstrasse 190, CH-8057 Zürich, Switzerland

E-mail: thomas.gehrmanneuzh.ch

\section{Nicola Adriano Lo Presti*}

Department of Physics, University of Zürich, Winterthurerstrasse 190, CH-8057 Zürich, Switzerland

E-mail: lopresti@physik.uzh.ch

\section{Johannes Henn}

PRISMA Cluster of Excellence, Johannes Gutenberg University, 55099 Mainz, Germany

E-mail: henn@uni-mainz.de

In these proceedings we present the computation of the full set of planar master integrals relevant to five-point amplitudes in massless QCD at two loops. We have applied these result to the fivegluon amplitude with all helicities positive in the leading-colours approximation, obtaining a very compact formula which, after subtracting terms related to the universal infrared and ultraviolet structure, consists only of dilogarithms.

Loops and Legs in Quantum Field Theory

24-29 April 2016

Leipzig, Germany

\footnotetext{
*Speaker.
} 


\section{Introduction}

In the LHC Run-II era, higher accuracy of theoretical predictions is needed more than ever. One-loop next-to-leading order (NLO) calculations have been established in the last decade as the new standard, even for very high-multiplicity processes, and they have achieved a high degree of automation, see e.g. [1, 2, 3, 4]. Two-loop next-to-next-to-leading order (NNLO) computations are the current frontier: while a plethora of two-to-two processes, with both colourless and colourful final states, have recently been computed, computations for two-to-three processes at the same accuracy still face major bottle-necks. Here we focus on the computation of the two-loop Feynman integrals which enter the purely virtual contribution of five-point massless cross sections at NNLO. While at one-loop all Feynman diagrams can be decomposed into a small sets of master integrals which are all known [5], at two-loop much larger set of master integrals appear, many of which remain to be calculated. We present here the calculation of the planar five-point Feynman integrals in the massless case $[6,7]$.

\section{Computation of the Feynman integrals}

Although it is possible to evaluate such integrals numerically through the sector decomposition approach $[8,9]$, these implementations tend not to be fast enough for the evaluation of the large number of points needed in a numerical simulation. For this and other reasons a fully analytic computation is needed.

In order to compute the required master integrals, we generate a system of first-order differential equations for these integrals by computing their derivatives with respect to external kinematic invariants [10] and by then reducing the resulting expressions to master integrals through integration-by-part identities $[13,14,15,16]$, a strategy which had been first applied to the case of four-point integrals with one external leg off-shell [11] needed in the $\gamma^{*} \rightarrow 3$ jet amplitude [12].

We define the massless five-point Feynman integral as

$$
\begin{aligned}
G_{a_{1}, \ldots, a_{11}} & =\int \frac{d^{D} k_{1} d^{D} k_{2}}{\left(i \pi^{D / 2}\right)^{2}} \frac{\left[-\left(k_{1}+p_{1}+p_{2}+p_{3}+p_{4}\right)^{2}\right]^{-a_{9}}}{\left[-k_{1}^{2}\right]^{a_{1}}\left[-\left(k_{1}+p_{1}\right)^{2}\right]^{a_{2}}\left[-\left(k_{1}+p_{1}+p_{2}\right)^{2}\right]^{a_{3}}\left[-\left(k_{1}+p_{1}+p_{2}+p_{3}\right)^{2}\right]^{a_{4}}} \\
& \frac{\left[-\left(k_{2}+p_{1}\right)^{2}\right]^{-a_{10}}\left[-\left(k_{2}+p_{1}+p_{2}\right)^{2}\right]^{-a_{11}}}{\left[-k_{2}^{2}\right]^{a_{5}}\left[-\left(k_{2}+p_{1}+p_{2}+p_{3}\right)^{2}\right]^{a_{6}}\left[-\left(k_{2}+p_{1}+p_{2}+p_{3}+p_{4}\right)^{2}\right]^{a_{7}}\left[-\left(k_{1}-k_{2}\right)^{2}\right]^{a_{8}}}
\end{aligned}
$$

with the five four-momenta on-shell, $p_{i}^{2}=0, i=1, \ldots, 5$, and all outgoing. We define the kinematic invatiants $v_{i}=2 p_{i} \cdot p_{i+1}$.

We find 61 master integrals, 17 of which correspond to genuine five-point topologies (of these only ten are independent, as the remaining seven can be obtained by cyclically permuting the external momenta). In parallel to our work, also another group has computed the five-point twoloop planar Feynman integrals, see [17].

As the basis of master integrals is not unique, it is possible to make choices which considerably simplify the differential equation. In particular, it was noted in [18] that it is possible to chose a basis of master integrals in such a way that the dimensional regulator $\varepsilon$ is factored out, i.e.

$$
\partial_{v_{i}} \vec{g}=A_{i}(\vec{v}, \varepsilon) \vec{g} \longrightarrow \partial_{v_{i}} \vec{f}=\varepsilon \tilde{A}_{i}(\vec{v}) \vec{f},
$$


which can be easily integrated order by order in $\varepsilon$.

The differential equation can be then further simplified and rewritten as

$$
d \vec{f}(\vec{v}, \varepsilon)=\varepsilon d\left[\sum_{k} a_{k} \log \alpha_{k}(\vec{v})\right] \vec{f}(\vec{v}, \varepsilon)
$$

where $a_{k}$ are constant matrices. The alphabet is

$$
\begin{aligned}
\vec{\alpha}=\{ & v_{1}, v_{2}, v_{3}, v_{4}, v_{5}, \\
& v_{1}-v_{4}, v_{2}-v_{5}, v_{3}-v_{1}, v_{4}-v_{2}, v_{5}-v_{3}, \\
& v_{1}+v_{2}-v_{4}, v_{2}+v_{3}-v_{5}, v_{3}+v_{4}-v_{1}, v_{4}+v_{5}-v_{2}, v_{5}+v_{1}-v_{3}, \\
& v_{3}+v_{4}, v_{4}+v_{5}, v_{5}+v_{1}, v_{1}+v_{2}, v_{2}+v_{3}, \\
& \left.r_{1}, r_{2}, r_{3}, r_{4}, r_{5}, \Delta\right\}
\end{aligned}
$$

with $r_{i}=\left(a_{i}+\sqrt{\Delta}\right) /\left(a_{i}-\sqrt{\Delta}\right), a_{i}=\operatorname{Cyclic}_{i}\left(v_{1} v_{2}-v_{2} v_{3}+v_{3} v_{4}-v_{1} v_{5}-v_{4} v_{5}\right)$, where 'Cyclic ${ }_{i}$ ' increases all indices of the argument of $i$, and $\Delta=\left|2 p_{i} \cdot p_{j}\right|(i, j=1, \ldots, 5)$.

The boundary values of the integrals are determined by requiring the cancellation of spurious singularities appearing in the differential equations, as it was done, for example, in [19]. An example is that, although no singularities appear in the Euclidean region $v_{i}<0$, the differential equation contains the letter $v_{1}+v_{2}-v_{4}$ which lead to spurious singularities when $v_{4}=v_{1}+v_{2}$. The requirement that, once the solution is plugged into the differential equation, these divergencies must cancel, allows us to constraint the value of the solution in these points, which then can be transported to a chosen reference point by solving the differential equation. In particular we also use the conditions imposed by the cancellation of spurious divergencies appearing on the $\Delta=0$ hypersurface. As a boundary point of reference we chose the symmetric point

$$
v_{i}=-1, \quad i=1, \ldots, 5 \text {. }
$$

An important consequence of eq. (2.2) is that the transcendental weight of the result, which is defined as the number of iterated integrations, is uniform at each order in $\varepsilon$. For the finite (i.e. $\left.\varepsilon^{0}\right)$ contribution to cross sections at two-loop accuracy, integration up to weight four is required. A very compact representation of the result can be obtained by direct integration of eq. (2.3) order by order in $\varepsilon$, leading to compact expressions in terms of the Chen-Iterated integrals [20,21].

\section{Results represented in terms of Goncharov polylogarithms}

Although a representation of the result in terms of Chen iterated integrals leads to very compact expressions and is well suited to algebraic manipulation, it is not ideal for its efficient numerical evaluation, for which a representation in terms of multiple polylogarithms [22] is better suited. By rewriting eq. (2.3) in such a way that only simple poles appear in the right-hand side, i.e.

$$
\partial_{x_{i}} \vec{f}=\varepsilon \sum_{k} \frac{\tilde{a}_{i, k}}{x_{i}-x_{i, k}} \vec{f},
$$



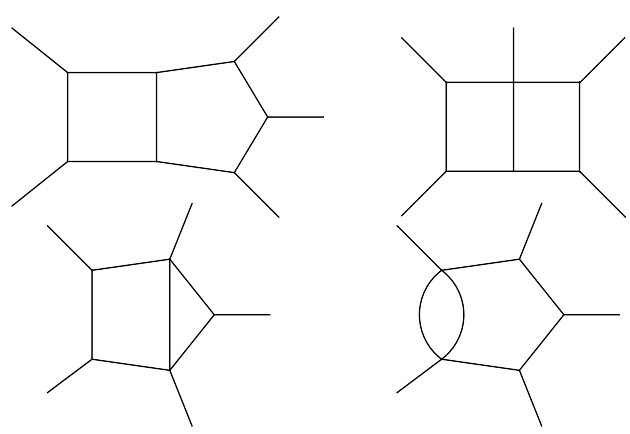

Figure 1: Genuine five-point planar two-loop topologies

the solution of the differential equation can be expressed in terms of Goncharov polylogarithms [23]

$$
\begin{array}{r}
G\left(a_{1}, a_{2}, \ldots, a_{n} ; x\right)=\int_{0}^{x} \frac{d t}{t-a_{1}} G\left(a_{2}, \ldots, a_{n} ; t\right), \\
\text { with } G(x)=1, \quad G(0)=0 \text { and } G\left(\overrightarrow{0}_{n} ; x\right)=\frac{1}{n !} \log ^{n} x \text {. }
\end{array}
$$

for which efficient numerical implementations exist [24]. This is achieved by parametrizing the kinematics using momemtum-twistor variables [25]; in particular we use

$$
\begin{aligned}
& v_{1} \rightarrow x_{1} \\
& v_{2} \rightarrow x_{1} x_{2} x_{4} \\
& v_{3} \rightarrow x_{1} \frac{x_{3}\left(x_{4}-1\right)+x_{2} x_{4}+x_{2} x_{3}\left(x_{4}-x_{5}\right)}{x_{2}} \\
& v_{4} \rightarrow x_{1} x_{2}\left(x_{4}-x_{5}\right) \\
& v_{5} \rightarrow x_{1} x_{3}\left(1-x_{5}\right)
\end{aligned}
$$

The inverse of this transformation is two-valued. We chose the inversion for which the symmetric point in eq. (2.5) is given by

$$
x_{1}=-1, x_{2}=\frac{1+\sqrt{5}}{2}, x_{3}=1, x_{4}=\frac{-1+\sqrt{5}}{2}, x_{5}=0 .
$$

This parametrization has the advantage of making the Gram determinant a perfect square, leading to

$$
\sqrt{\Delta}=-x_{1}^{2}\left[x_{2} x_{4}\left(x_{5}-1\right)+x_{3}\left(1+x_{2} x_{5}+x_{4}\left(-2-x_{2}+x_{5}\right)\right)\right]
$$

(the overall sign is chosen in such a way that $\sqrt{\Delta}$ is positive in the symmetric point (3.4)).

The differential equations are then integrated for all 61 master integrals one variable at a time. In principle, even if the result does not contain square roots originating from the Gram determinant, additional square roots may arise from partial fractioning quadratic denominators to obtain eq. (3.1). This puts some constraint on the ordering of the integration variables. Choosing $x_{2}$ as the 
first variable to be integrated leads only to square roots of numbers, which are no problem, since all other variables are kept fixed. Also, it is convenient to integrate $x_{1}$ last, since the differential equation matrix with respect to this variable has the particularly simple form $\operatorname{diag}\left(-2 / x_{1}, \ldots,-2 / x_{1}\right)$. The integration path is chosen as a polygonal chain formed by segments which are parallel to the $x_{i}$ axes, in the order $\left\{x_{2}, x_{5}, x_{3}, x_{4}, x_{1}\right\}$.

The four-point sub-topologies have been compared numerically with the results of [11] up to weight 4 , finding perfect agreement. All integrals have been validated against values obtained through numerical integration [9] in the Euclidean region $v_{i}<0, i=1, \ldots, 5$.

\section{Result for the five-gluon all-plus amplitude}

The reduction of two-loop scattering amplitudes to a set of master integrals is still an outstanding problem. For five-point massless QCD amplitudes, the reduction to a basis of integrals was achieved in the case of external legs having all helicities positive [26, 27]. The integrals we have computed allowed us to obtain the fully analytic expression of this amplitude in the leadingcolour approximation

$$
\begin{gathered}
\left.\mathscr{A}_{5}\left(1^{+} 2^{+} 3^{+} 4^{+} 5^{+}\right)\right|_{\text {leading color }}=g^{3} \sum_{L \geq 1}\left(g^{2} N c_{\Gamma}\right)^{L} \\
\times \sum_{\sigma \in S_{5} / Z_{5}} \operatorname{tr}\left(T^{a_{\sigma(1)}} T^{a_{\sigma(2)}} T^{a_{\sigma(3)}} T^{a_{\sigma(4)}} T^{a_{\sigma(5)}}\right) \\
\times A_{5}^{(L)}\left(\sigma(1)^{+} \sigma(2)^{+} \sigma(3)^{+} \sigma(4)^{+} \sigma(5)^{+}\right),
\end{gathered}
$$

where $S_{5} / Z_{5}$ denote all non-cyclic permutations of five points. This amplitude vanishes at tree-level and is therefore finite at one loop,

$$
A_{5}^{(1)}=R F_{5}^{(1)}+\mathscr{O}(\varepsilon)
$$

with $R=i / 6 /(\langle 12\rangle\langle 23\rangle\langle 34\rangle\langle 45\rangle\langle 51\rangle)$ and [28]

$$
F_{5}^{(1)}=v_{1} v_{2}+v_{2} v_{3}+v_{3} v_{4}+v_{4} v_{5}+v_{5} v_{1}+\operatorname{tr}_{5}
$$

At two loops, the infrared and ultraviolet divergent terms can be predicted in terms of the one-loop result [29]

$$
A_{5}^{(2)}=A_{5}^{(1)}\left[-\sum_{i=1}^{5} \frac{1}{\varepsilon^{2}}\left(\frac{\mu^{2}}{-v_{i}}\right)^{\varepsilon}\right]+R F_{5}^{(2)}+\mathscr{O}(\varepsilon),
$$

In order to define the finite reminder $F_{5}^{(2)}$, the one-loop amplitude $A_{5}^{(1)}$ in the previous equation needs to be expanded up to order $\varepsilon^{2}$. This involves the computation of the one-loop massless pentagon integral up to that order in $\varepsilon$, which we obtained using the same method that we have used for the determination of the two-loop integrals. The fact that the double and single poles in $\varepsilon$ of eq. (4.4) are correctly reproduced provides an additional non-trivial check of the correctness of our results up to weight three. In the finite remainder, all functions of weight one, three and four cancel out and we can express the remaining weight-two functions in terms of dilogarithms, 
finding the following expression [6],

$$
\begin{gathered}
F_{5}^{(2)}=\frac{5 \pi^{2}}{12} F_{5}^{(1)}+\sum_{i=0}^{4} \sigma^{i}\left\{\frac{v_{5} \operatorname{tr}\left[\left(1-\gamma_{5}\right) \not \phi_{4} \not \phi_{5} \not \not_{1} \not_{2}\right]}{\left(v_{2}+v_{3}-v_{5}\right)} I_{23,5}\right. \\
\left.+\frac{1}{6} \frac{\operatorname{tr}\left[\left(1+\gamma_{5}\right) \not \not_{4} \not \not_{5} \not \phi_{1} \not \phi_{2}\right]^{2}}{v_{1} v_{4}}+\frac{10}{3} v_{1} v_{2}+\frac{2}{3} v_{1} v_{3}\right\} .
\end{gathered}
$$

where $\sigma^{i}$ cyclically shifts all indices (of $p, v$, and $I$ ) by $i$, and where

$$
I_{23,5}=\zeta_{2}+\operatorname{Li}_{2}\left[\frac{\left(v_{5}-v_{2}\right)\left(v_{5}-v_{3}\right)}{v_{2} v_{3}}\right]-\mathrm{Li}_{2}\left[\frac{v_{5}-v_{3}}{v_{2}}\right]-\mathrm{Li}_{2}\left[\frac{v_{5}-v_{2}}{v_{3}}\right] .
$$

Note that eq. (4.5) contains both parity odd and even terms. We have checked our result via direct numerical comparison with [26]. We have also checked eq. (4.5) in the limit of two external legs being collinear, where the amplitude is expected to factorize as the sum of products of splitting amplitudes [30] and lower-point amplitudes [31]. Eq. (4.5) was also confirmed independently in [32] following a completely different approach based on generalized unitarity.

\section{Conclusions}

In these proceedings we have presented our recent calculation of the full set of two-loop planar master integrals entering massless five-point QCD amplitudes [7] using the method of differential equations $[10,18]$. Our result provides the complete set of functions which can appear in a generic planar massless five-particle scattering amplitude at two loops, reducing the calculation of any such scattering amplitude to the determination of the algebraic coefficients, see e.g. [26, 35], accompanying the integral basis.

We have applied the integrals that we have computed to the two-loop five-gluon amplitude with all helicities positive in the leading-colour approximation, obtaining a very compact analytic result [6]. Given the compactness of the analytic result for the all-plus amplitude, this result will also give useful hints for generalizations for any number of external legs, see recent revelopments in $[34,33]$.

An important next step will be the computation of the master integrals corresponding to nonplanar topologies, which, for instance, are required for the full-colour all-plus amplitude [27].

\section{Acknowledgements}

This work was supported in part by the Schweizer Nationalfonds under grant 200020-162487, as well as by the European Commission through the ERC Advanced Grant "MC@NNLO" (340983). J.M.H. is supported in part by a GFK fellowship and by the PRISMA cluster of excellence at Mainz university. 


\section{References}

[1] C. F. Berger, Z. Bern, L. J. Dixon, F. Febres Cordero, D. Forde, H. Ita, D. A. Kosower and D. Maitre, Phys. Rev. D 78 (2008) 036003

[2] G. Cullen, N. Greiner, G. Heinrich, G. Luisoni, P. Mastrolia, G. Ossola, T. Reiter and F. Tramontano, Eur. Phys. J. C 72 (2012) 1889

[3] S. Badger, B. Biedermann, P. Uwer and V. Yundin, Comput. Phys. Commun. 184 (2013) 1981

[4] F. Cascioli et al., Phys. Lett. B 735 (2014) 311

[5] R. K. Ellis and G. Zanderighi, JHEP 0802 (2008) 002

[6] T. Gehrmann, J. M. Henn and N. A. Lo Presti, Phys. Rev. Lett. 116 (2016) 062001 Erratum: [Phys. Rev. Lett. 116 (2016) 189903] [arXiv:1511.05409 [hep-ph]].

[7] T. Gehrmann, J.M. Henn and A. Lo Presti, in preparation.

[8] G. Heinrich, Int. J. Mod. Phys. A 23 (2008) 1457; S. Borowka, G. Heinrich, S. P. Jones, M. Kerner, J. Schlenk and T. Zirke, Comput. Phys. Commun. 196 (2015) 470.

[9] A. V. Smirnov, V. A. Smirnov and M. Tentyukov, Comput. Phys. Commun. 182 (2011) 790; A. V. Smirnov, Comput. Phys. Commun. 185 (2014) 2090.

[10] T. Gehrmann and E. Remiddi, Nucl. Phys. B 580 (2000) 485.

[11] T. Gehrmann and E. Remiddi, Nucl. Phys. B 601 (2001) 248. Nucl. Phys. B 601 (2001) 287.

[12] L. W. Garland, T. Gehrmann, E. W. N. Glover, A. Koukoutsakis and E. Remiddi, Nucl. Phys. B 627 (2002) 107; Nucl. Phys. B 642 (2002) 227.

[13] F. V. Tkachov, Phys. Lett. B 100 (1981) 65; K. G. Chetyrkin and F. V. Tkachov, Nucl. Phys. B 192 (1981) 159.

[14] S. Laporta, Int. J. Mod. Phys. A 15 (2000) 5087.

[15] A. V. Smirnov, JHEP 0810 (2008) 107; A. V. Smirnov, Comput. Phys. Commun. 185, 2090 (2014).

[16] C. Studerus, Comput. Phys. Commun. 181 (2010) 1293; A. von Manteuffel and C. Studerus, arXiv:1201.4330 [hep-ph].

[17] C. G. Papadopoulos, D. Tommasini and C. Wever, JHEP 1604 (2016) 078

[18] J. M. Henn, Phys. Rev. Lett. 110 (2013) 251601.

[19] J. M. Henn, A. V. Smirnov and V. A. Smirnov, JHEP 1403, 088 (2014).

[20] Kuo-Tsai Chen, Bull. Amer. Math. Soc. Volume 83, Number 5 (1977), 831-879.

[21] S. Caron-Huot and J. M. Henn, JHEP 1406, 114 (2014).

[22] E. Remiddi and J. A. M. Vermaseren, Int. J. Mod. Phys. A 15 (2000) 725.

[23] A. B. Goncharov, Math. Res. Lett. 5 (1998) 497.

[24] J. Vollinga and S. Weinzierl, Comput. Phys. Commun. 167 (2005) 177.

[25] A. Hodges, JHEP 1305, 135 (2013); L. J. Mason and D. Skinner, JHEP 0911, 045 (2009).

[26] S. Badger, H. Frellesvig and Y. Zhang, JHEP 1312 (2013) 045;

[27] S. Badger, G. Mogull, A. Ochirov and D. O'Connell, JHEP 1510 (2015) 064. 
[28] Z. Bern, L. J. Dixon, D. C. Dunbar and D. A. Kosower, Nucl. Phys. B 425, 217 (1994).

[29] S. Catani, Phys. Lett. B 427, 161 (1998).

[30] D. A. Kosower and P. Uwer, Nucl. Phys. B 563 (1999) 477; Z. Bern, V. Del Duca, W. B. Kilgore and C. R. Schmidt, Phys. Rev. D 60 (1999) 116001; Z. Bern, L. J. Dixon and D. A. Kosower, JHEP 0408 (2004) 012; S. D. Badger and E. W. N. Glover, JHEP 0407 (2004) 040.

[31] Z. Bern, A. De Freitas and L. J. Dixon, JHEP 0203, 018 (2002).

[32] D. C. Dunbar and W. B. Perkins, Phys. Rev. D 93 (2016) no.8, 085029 [arXiv:1603.07514 [hep-th]].

[33] D. C. Dunbar, G. R. Jehu and W. B. Perkins, Phys. Rev. D 93 (2016) no.12, 125006 [arXiv:1604.06631 [hep-th]].

[34] D. C. Dunbar, G. R. Jehu and W. B. Perkins, arXiv:1605.06351 [hep-th].

[35] G. Ossola, C. G. Papadopoulos and R. Pittau, Nucl. Phys. B 763, 147 (2007). 